\title{
ANGIOGENESIS' RELATED POLYMORPHISM EMERGES AS PROGNOSTIC MARKER IN DE NOVO LARGE B-CELL LYMPHOMA PATIENTS
}

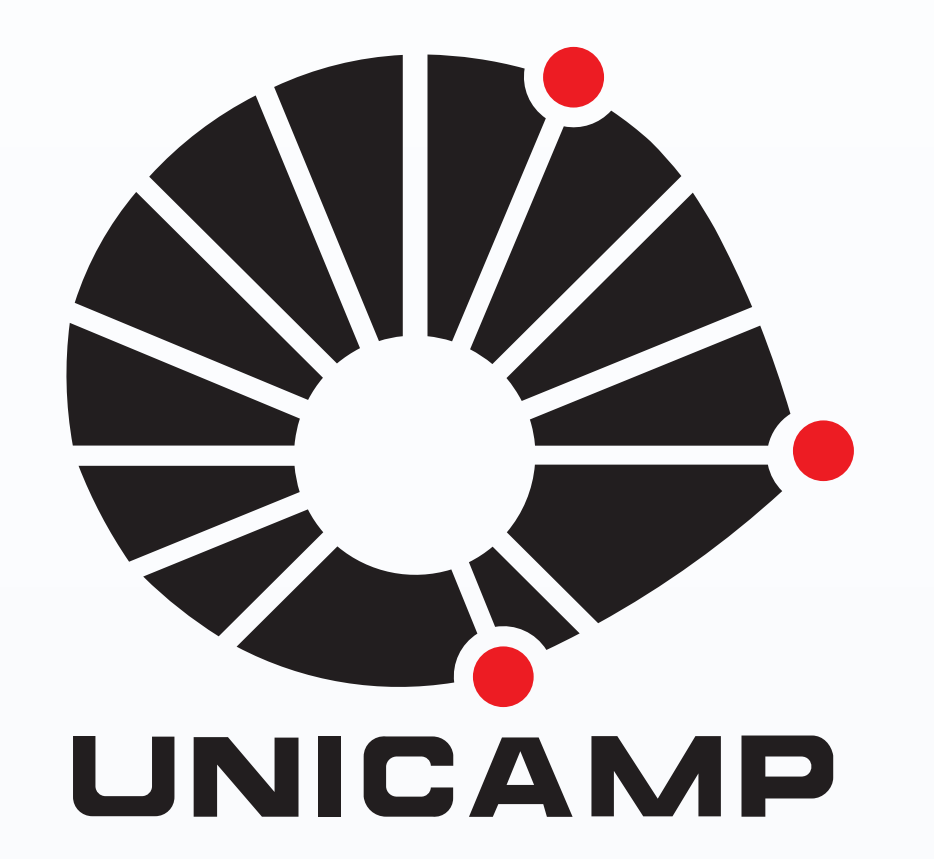

Angelo Borsarelli Carvalho Brito', Marcia Torresan Delamain', Cármino Antônio de Souza, , José Vassallo ${ }^{3,4}$, Carmen Silvia Passos Lima'

${ }^{1}$ Department of Internal Medicine, Faculty of Medical Sciences, University of Campinas, Campinas, São Paulo, Brazil

${ }^{2}$ Hematology and Hemotherapy Center, University of Campinas, Campinas, São Paulo, Brazil

A. C. Camargo Cancer Center, São Paulo, São Paulo, Brazil

${ }^{4}$ Laboratory of Molecular and Investigative Pathology, Faculty of Medical Sciences, University of Campinas, Campinas, São Paulo, Brazil,

School of Medical Sciences, University of Campinas, Campinas, Brazil

KEY WORDS: Lymphoma, genetic polymorphism, angiogenesis

BACKGROUND

Angiogenesis ( $A G$ ), the process in which new vessels are formed from preexisting vasculature, is
crucial for the growth and progression of diffuse large $B$ cell lymphoma (DLBCL) [1]. The most important key regulator of $A G$ is the vascular endohelial growth factor (VEGF), and its functions is polymorphic genes and single nucleotide polymorphisms (SNPs) determining are encoded by polymorphic genes and single nuclessions have been described.

One of the main problems hindering the therapeutic success in DLBCL patients is the heterogeneity of this tumor, not only in clinical and morphologic level but also in the genetic level [3]. Immunohistochemistry (IHC) based on the expression of proteins that are differentially However, patients who present the same clinical features, when stratified by the Ann $A$, 5 , System, R-IPI or cell of origin IHC-based algorithm, may present distinct behavior, what suggests that new prognostic (genetic and IHQ markers) remains to be identified in disease.

Once that the association of VEGF 2578C/A (rs699947), 2489C/T (rs1005230), 1154G/A (rst57036), $344 \mathrm{G} / \mathrm{C}$ (rs2010963), 406C/T (rs833061) and 936C/T (rs3025039) SNPs, an (rs1870377) SNPs in tumor vasculature VEGFNEGFR2 expression, clinical and 1719A/T survival of DLBCL patients is not yet clarified, the aim of this study was defined.

SUBJECTS AND METHODS

This prospective study included 168 consecutive de novo DLBCL patients seen at diagnosis at the Haematology and Haemotherapy Centre and A. C. Camargo Cancer Centre from June 2009 to Arbor critria. All DaBiel was don cyclophoshamide doxorubicin vincristine, and prednison (R-CHOP) [6] All procedures were carried out according to the principles of the local ethics committee, and all subjects provided written informed consent.

Immunohistochemical analysis

Patients' diagnostic paraffin blocks served for IHC analyses using anti-CD34, anti-VEGF and anti(Aperio Technologies, Vista, CA USA) and submitted for digital analysis in a blinded fashion analyzed. VEGF was analyzed by the intensity of positivity (positive-stained pixels) and VEGFR2 was categorized in negative and positive cases.

Genetic polymorphism analysis

DNA from patients' peripheral blood samples was analyzed by real-time polymerase chain (Carlsbad CA USA) forVE TaqMan SNP Genotyping Assays from Applied Blosystems and VEGFR2 271G/A, 604T/C, $1192 \mathrm{G} / \mathrm{A}$ and $1719 \mathrm{~A} / \mathrm{ANPS}$. All reactions were performs according to the manufacturers' instructions. The amount of $15 \%$ of genotype determinations was carried out twice in independent experiments with $100 \%$ of concordance.

Statistical analysis

The Hardy-Weinberg equilibrium (HWE) was evaluated using the chi-square $\left(\chi^{2}\right)$ goodness-of-fit hest. Haploview 4.2 sofware (wWW.broad.mit.edu/mpg haploview) was used o tag all com $10 \%$ were selected to analysis.

Differences between groups were analyzed by the $\chi^{2}$ or Fisher's exact test. Two-tailed t-tests were scores between groups of patients. Logistic

Event-free survival (EFS) and overall survival (OS) were calculated from the date of diagnosis to date of progression, relapse, death by disease or last follow-up, and from the date of diagnosis to used to identify variables predicting EFS and $O S$. Variables with $P<0.10$ in univariate Cox analysis were included in multivariate Cox analysis. Significant results were validated using a bootstrap significantwhen $\mathrm{P}<0.05$.
RESULTS

(The median of observation of patients enrolled in the study was 51 months (range: 1-117 months).

Concerning AG's SNPS and clinical features, VEGF 634GG was more frequent in more common in those who presented grade III or IV toxicities during treatmas more common in those who presented grade 111 or IV toxicities during treatment
$(21.6 \%$ vs. $8.5 \%, P=0.03)$ when compared to others. VEGGF $936 C T$ or TT genotypes 政 vs. $22.6 \%, P=0.01$ ) and was more common in patients with III or IV toxicity during treatment $(41.2 \%$ vs. $19.8 \%, P=0.007)$ when compared to others. VEGFR2 $604 T \mathrm{TT}$
was more frequent in poor $\mathrm{R}-\mathrm{IPI}$ patients $(43.7 \%$ vs. $27.8 \%, \mathrm{P}=0.04)$ when was more frequen
compared to others.

Concerning IHQ analysis, VEGF 634G allele was associated with higher vesse area and vascular perimeter; VEGF 936T allele was associated with VEGFR2 expression and VEGFR2 1719AA genotype was associated with VEGF expression. The prognostic impact of VEGF $1154 \mathrm{G} / \mathrm{A}$ SNP and VEGF ATCAGC haplotype is
represented in Kaplan Meier curve (Figure 1) and Cox analysis (Table 2).

\begin{tabular}{|c|c|}
\hline Variable & $\mathrm{N}(\%)$ \\
\hline \multicolumn{2}{|l|}{ Age } \\
\hline \multicolumn{2}{|l|}{ Gender } \\
\hline Female & $94(55.9)$ \\
\hline Male & $74(44.1)$ \\
\hline \multicolumn{2}{|l|}{ B symptoms } \\
\hline Absent & $49(29.2)$ \\
\hline Present & $119(70.8)$ \\
\hline \multicolumn{2}{|l|}{ Bulky disease } \\
\hline No & $111(66.1)$ \\
\hline Yes & $57(33.9)$ \\
\hline \multicolumn{2}{|l|}{ Ann Arbor } \\
\hline I+II & $74(44,1)$ \\
\hline $\mathrm{III}+\mathrm{IV}$ & $94(55.9)$ \\
\hline \multicolumn{2}{|l|}{ R-IPI } \\
\hline Very good/good & $97(57.7)$ \\
\hline Poor & $71(42.3)$ \\
\hline \multicolumn{2}{|l|}{ IHC-based subtype* } \\
\hline $\mathrm{GCB}$ & $88(71.5)$ \\
\hline Non-GCB & $35(28.5)$ \\
\hline \multicolumn{2}{|l|}{ Response to R-CHOP*** } \\
\hline Complete & $115(83.3)$ \\
\hline Partial & $7(5.1)$ \\
\hline Stable or progressive disease & $16(11.6)$ \\
\hline \multicolumn{2}{|l|}{ Toxicity grade III or IV*** } \\
\hline Haematological & $49(96.1)$ \\
\hline Non-hematological & $2(3.9)$ \\
\hline
\end{tabular}

Table 2. Association of clinicopathological features and VEGF and VEGFR2 genotypes with survival in diffuse large B-cell lymphoma patients

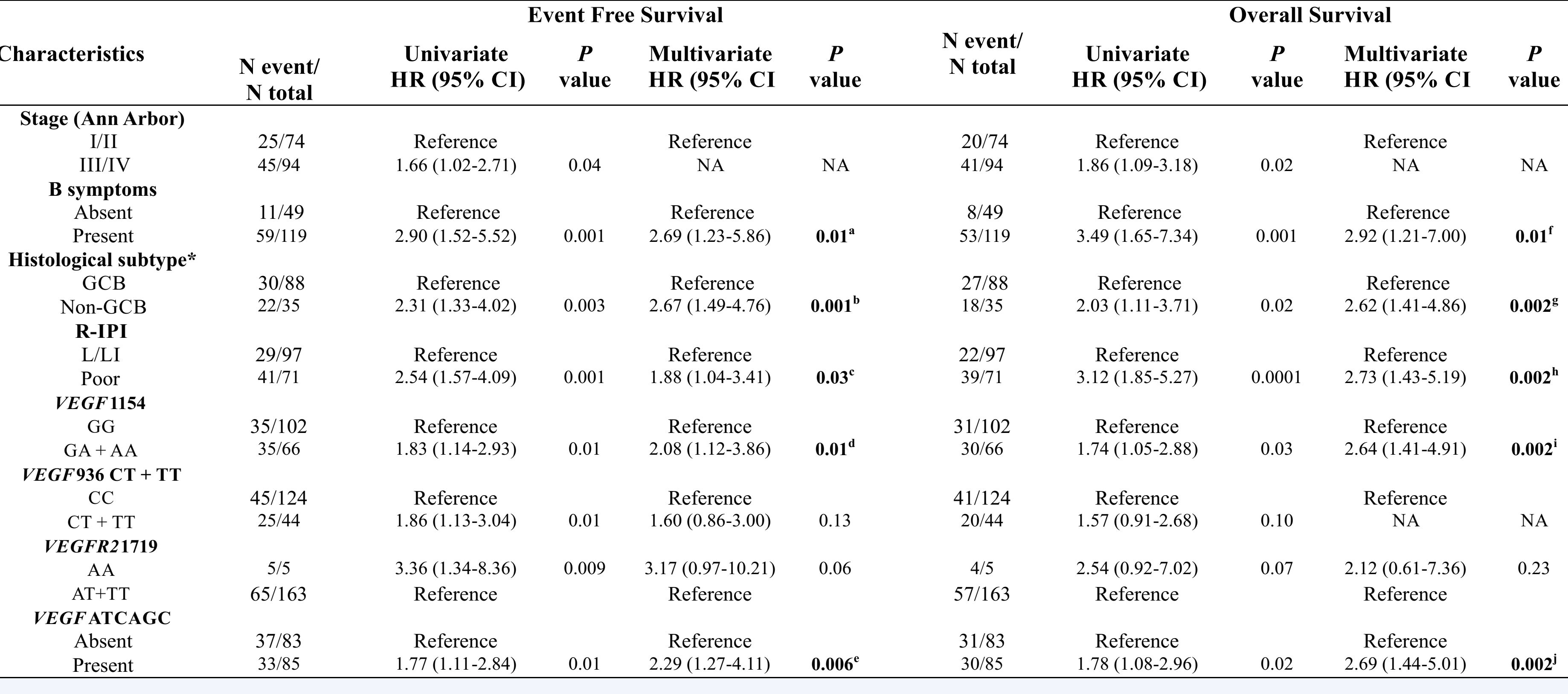

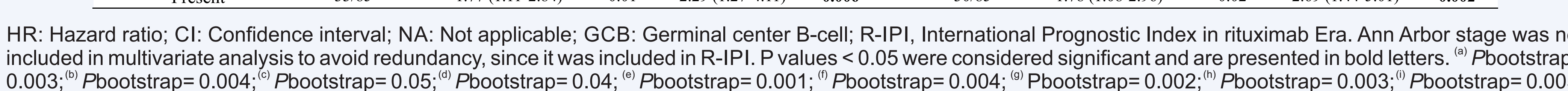
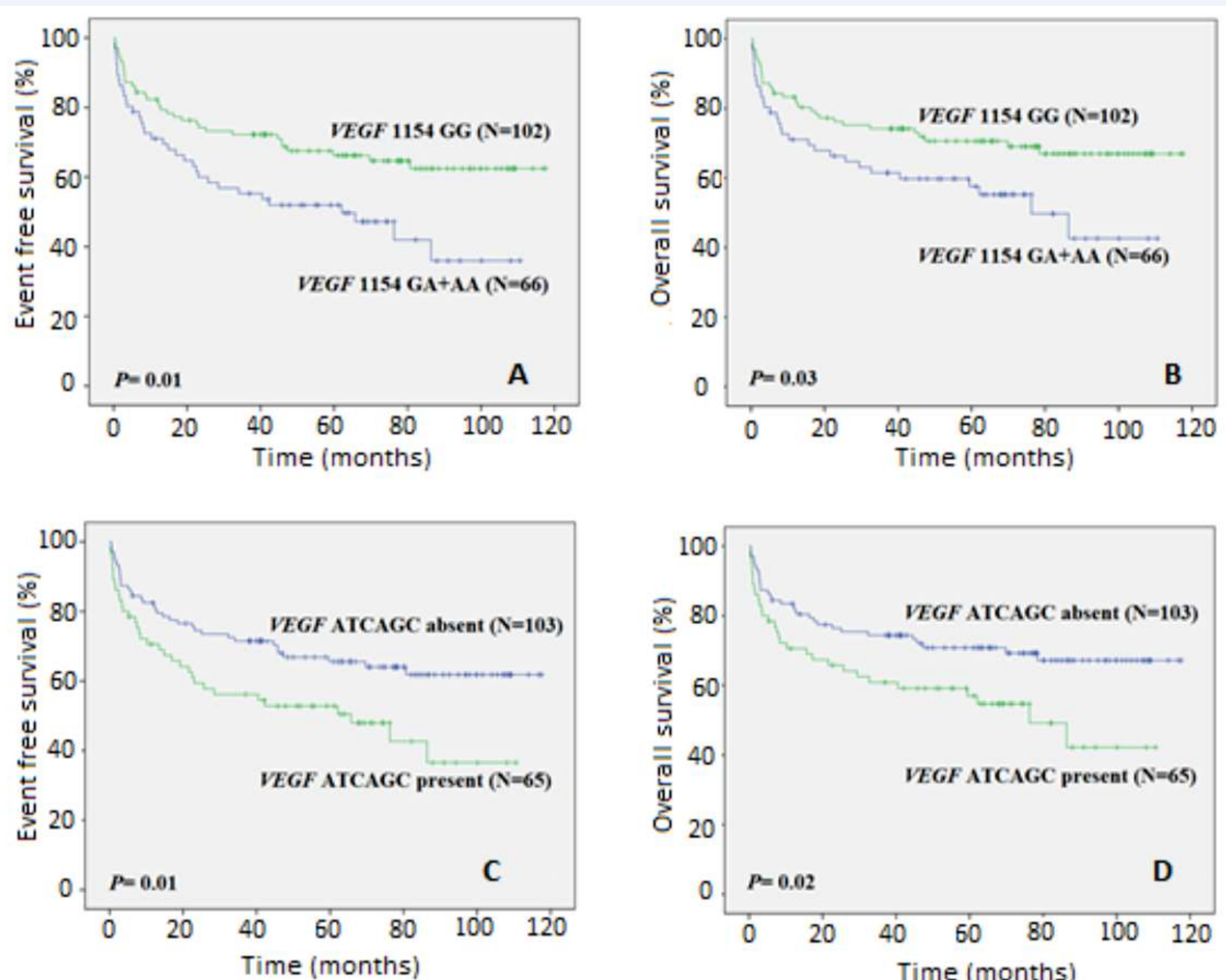

Figure 1. a) Event-free survival (EFS) and b) overall survival (OS) of diffuse large B-cell lymphoma (DLBCL) DLBCL patients stratified by the presence VEGFATCAGC haplotype

CONCLUSIONS

In conclusion, our data present evidence that inherited abnormalities in VEGF and VEGFR2 SNPs, enrolled in arger sample size and by functional analyses of SNPs in tumor AG.

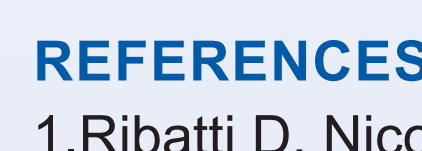

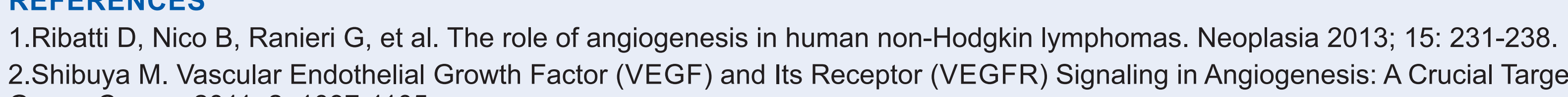

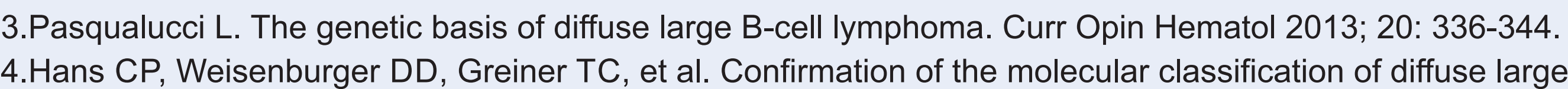

